\title{
A Case Report of Partial Atrio Ventricular Canal on A 28 Years old Patient
}

\author{
Henry Mayala*, Pedro Pallangyo, Khamis Hassan Bakari, Fabian Pius \\ Mghanga, Mohamed Janabi, Wang Zhao Hui \\ Jakaya Kikwete Cardiac Institute, Tongji Medical College Of Huazhong University Of Science And Technology
}

\begin{abstract}
Background: Partial AV canal defect is a form of endocardial cushion defect infrequently encountered in Adults. It includes an ostium primum atrial septal defect but with two separate atrioventricular valve rings and a cleft in the anterior Mitral valve leaflet.

Case Report: We present a case report of 28 years old female, who attended at outpatient department of Jakaya Kikwete Cardiac Institute with the presenting complains of difficulty in breathing on exertion and awareness of heart beat. Clinical and imaging findings are suggestive of partial AV canal defect.

Conclusion: Despite atrial septal defect being the third most common form of congenital heart disease in adult patients, we thought the importance of understanding the natural history and clinical significance for its diagnosis, as it may go un detected for some patients making it necessary for this case report.
\end{abstract}

Keywords: AV-canal-atrio ventricular canal, ostium primum atrial septal defect

\section{Introduction}

Atrioventricular septal defects (AVSDs) are anatomic defects that arise from faulty development of the embryonic endocardial cushions. This spectrum ranges from a primum atrial septal defect and cleft mitral valve, known as a partial atrioventricular septal defect (partial AVSD), to defects of both the primum atrial septum and inlet ventricular septum and the presence of a common atrioventricular valve, referred to as complete atrioventricular septal defect (complete AVSD, CAVSD). The terms atrioventricular canal defect and endocardial cushion defect are used in reference to this group of defects; however, atrioventricular septal defect is now the preferred terminology. These defects, particularly the complete form, typically present in the fetal or neonatal period and are an important source of cardiac morbidity and mortality in this age group. ${ }^{1}$

Partial AV canal defect is a form of endocardial cushion defect infrequently encountered in Adults. It includes an ostium primum atrial septal defect but with two separate atrioventricular valve rings and a cleft in the anterior Mitral valve leaflet.Diagnosis of CAVC might be clinically suspected in patients presenting in the first few months of life with congestive heart failure, cardiomegaly on chest X-ray and left axis deviation, biatrial enlargement and bi-ventricular pressure and volume overload on electrocardiogram (ECG). Echocardiography is the key tool for the diagnosis and anatomic classification of this malformation. It shows the ostium primum atrial septal defect, with the underlying common atrioventricular valve, and the defect of the ventricular septal inflow.Atrioventricular Septal defect can be classified as: Complete and Partial Atrioventricular septal defect respectively. Half of children with untreated CAVC die in the first year of life. The main cause of death in infancy is either heart failure or pneumonia. In surviving patients with unrepaired complete atrioventricular canal, irreversible pulmonary vascular disease becomes increasingly common, and affects virtually all patients older than 2 years of age. Long-term prognosis in patients with irreversible pulmonary hypertension is poor. ${ }^{3}$

\section{Case Presentation}

We present a case report of a 28 years old female, who attended at outpatient department of Jakaya Kikwete Cardiac Institute of Muhimbili national hospital with the presenting complains of difficulty in breathing on exertion and awareness of heart beat, on physical examination she has a apical systolic murmur. The patient underwent the following investigations: Chest x-ray, ECG, and Echocardiography their findings were as follows:

Chest X-ray- was normal, with normal cardiac silhouette, normal lungs fields

Figure: 1; A Chest $\mathrm{x}$-ray (PA-view) revealing a normal chest $\mathrm{x}$-ray findings

ECG: revealed features of right bundle branch block

Figure: 2; ECG showing right bundle branch block 
Echocardiography revealed a single atrium, mitral valve and tricuspid valve well located, with a cleft on the anterior mitral valve leaflet; there was mild Mitral and tricuspid regurgitation, preserved LV systolic function $\mathrm{EF}=60 \%$, no thrombus seen. Normal pericardium

Figure: 3; the Apical four chamber view showing a single atrium.

Figure: 4; a parasternal long axis view showing a cleft on the anterior mitral valve leaflet

Figure: 5; Apical four chamber showing an ostium primum atrial septal defect but with two separate atrioventricular valve rings

\section{Discussion}

As explained earlier Partial AV canal frequently encountered in early childhood due embryogenic defects, but sometimes it can go undetected without symptoms up to adult hood and present with symptoms, like in case of our patient the symptoms presented for the first time when he visited our hospital, presenting with awareness of heart beat, difficulty in breathing on exertion, upon doing physical examination and further investigations a diagnosis of Partial AV canal was attained, the patient was then managed clinically by lasix, aldactone, bisoprolol and enalapril, inorder to stabilize her and scheduled for surgical repair upon stabilization. Awareness and understanding of the condition is critical and might determine the early management and prevent complications, furthermore, the most import thing is post operative care after the repair. We thought the importance of understanding the natural history and clinical significance for its diagnosis, as it may go un detected for some patients making it necessary for this case report.

We declare there is no conflict of interest

[1]. http://emedicine.medscape.com/article/893914-overview

[2]. http://circ.ahajournals.org/content/66/2/284.full.pdf

[3]. http://www.ncbi.nlm.nih.gov/pmc/articles/PMC1459121/

[4]. Wiener L, Schneider PJ, Cox JW: Persistent cardiac ostium primum: a diagnostic dilemma in the aged. J Am Geriatr Soc 14: 1156, 1966

[5]. Campbell M, Neill C, Suzman S: The prognosis of atrial septal defect. Br Med J 1: 1375, 1957

[6]. Heath D: Long survival in partial persistent common atrioventricular canal. Br J Dis Chest 62: 207, 1968

[7]. Fontana RS, Edwards JE: Congenital Cardiac Disease: A Review of 357 Cases Studied Pathologically. Philadelphia, WB Saunders, 1962

[8]. Weyn AS, Bartle SH, Nolan TB, Dammann JF Jr: Atrial septal defect - primum type. Circulation 32 (suppl III): III-13, 1965

[9]. Losay J, Rosenthal A, Castaneda AR, Bernhard WH, Nadas AS: Repair of atrial septal defect primum: results, course,and prognosis. J Thorac Cardiovasc Surg 75: 248, 1978

[10]. McMullan MH, McGoon DC, Wallace RB, Danielson GK, Weidman WH: Surgical treatment of partial atrioventricular canal. Arch Surg 107: 705, 1973

[11]. Hagler DJ, Tajik AJ, Seward JB, Mair DD, Ritter DG: Real-time wide-angle sector echocardiography: atrioventricular canal defects. Circulation 59: 140, 1979

[12]. Fuster V, Brandenburg RO, McGoon DC, Giuliani ER: Clinical approach and management of congenital heart disease in the adolescent and adult. Cardiovasc Clin 10: 161, 1980

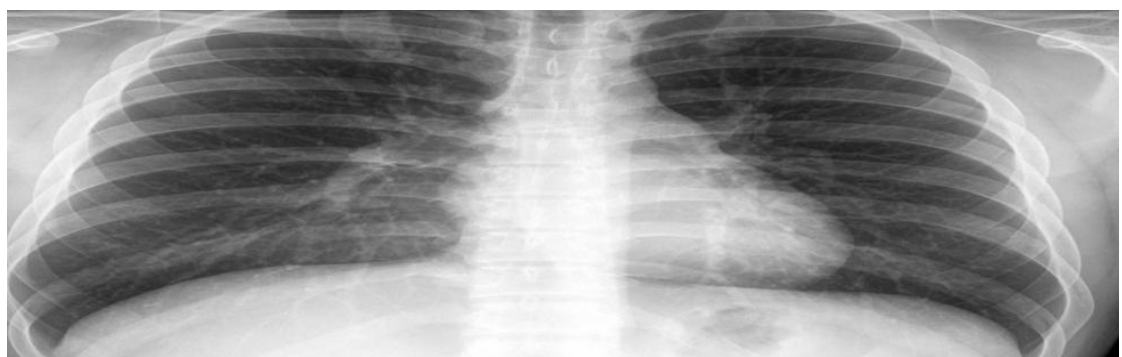

Figure 1: A Chest X-ray (PA-view) revealing a normal chest $\mathrm{x}$-ray findings

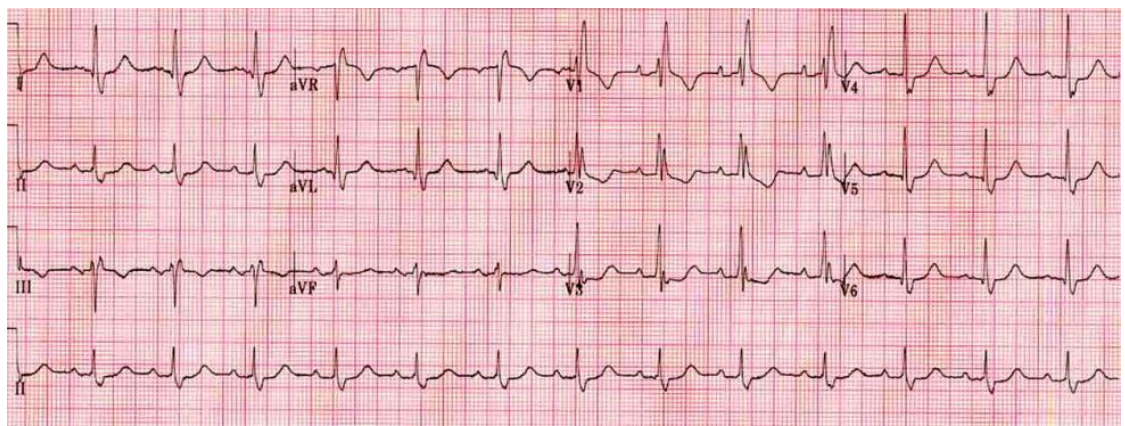

Figure 2: ECG showing right bundle branch block 


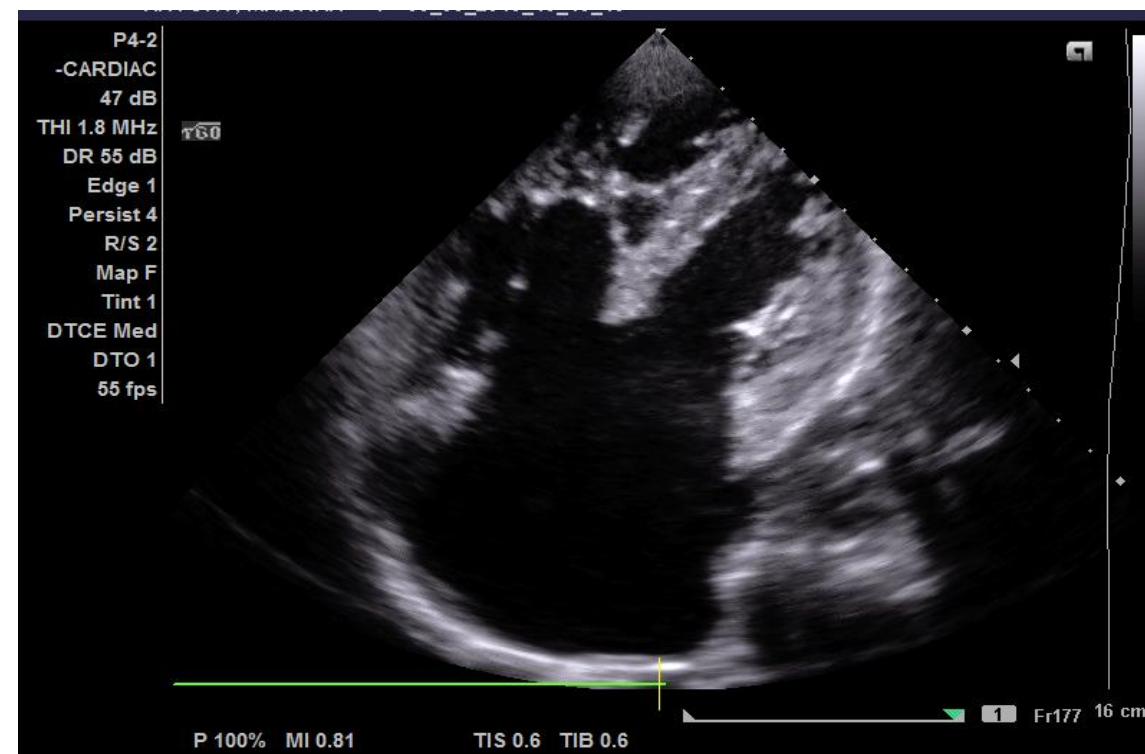

Figure 3: the Apical four chamber view showing a single atrium.

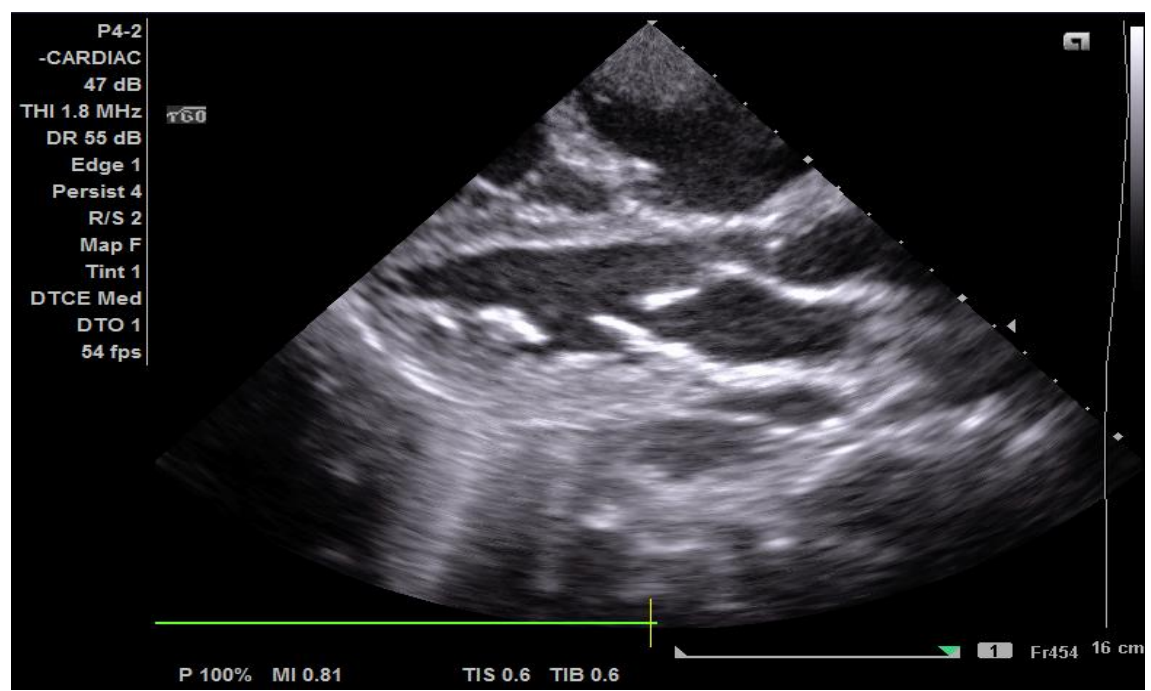

Figure 4: A parasternal long axis view showing a cleft on the anterior mitral valve leaflet

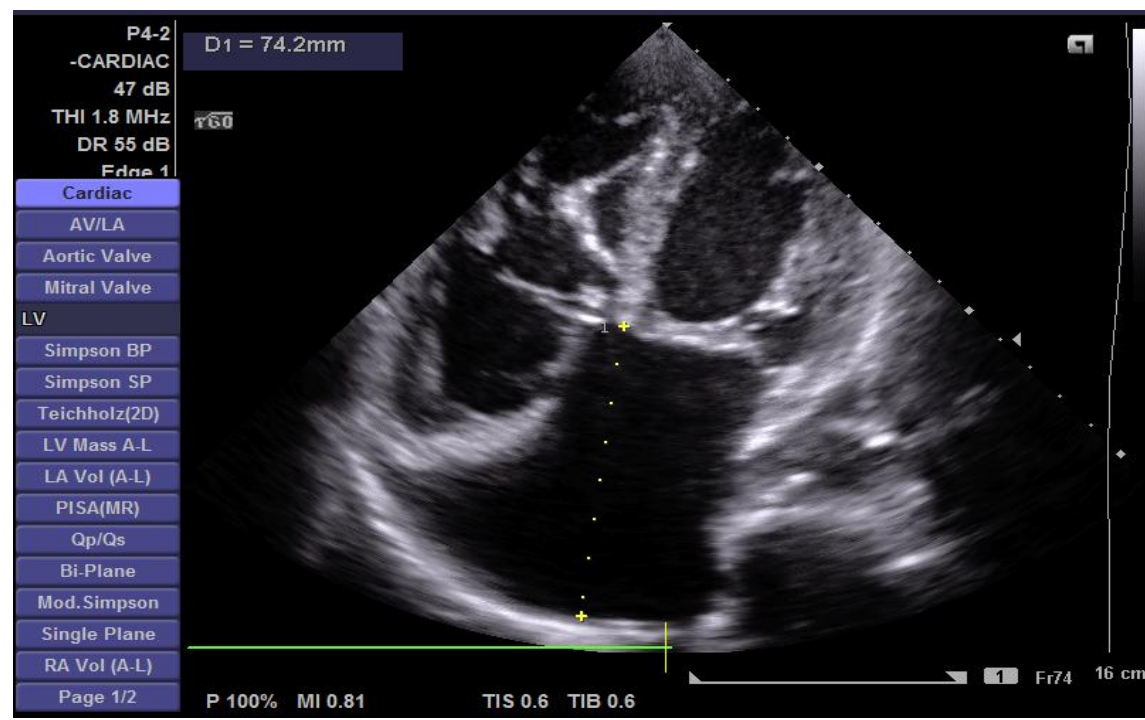

Figure 5: Apical four chamber showing an ostium primum atrial septal defect but with two separate atrioventricular valve rings 